\title{
Therapeutic or spontaneous Helicobacter pylori eradication can obscure magnifying narrow-band imaging of gastric tumors
}

Authors

Institutions
Masaaki Kobayashi', Satoru Hashimoto ${ }^{2}$, Ken-ichi Mizuno², Manabu Takeuchi², Yuichi Sato ${ }^{2}$, Gen Watanabe ${ }^{3}$, Yoichi Ajioka ${ }^{3}$, Motoi Azumi ${ }^{2,4}$, Kouhei Akazawa ${ }^{4}$, Shuji Terai ${ }^{2}$

Institutions are listed at the end of article. submitted

25. September 2015 accepted after revision 21. March 2016

\section{Bibliography}

DOI http://dx.doi.org/

10.1055/s-0042-105869

Published online: 12.5.2016

Endoscopy International Open

2016; 04: E665-E672

(c) Georg Thieme Verlag KG

Stuttgart - New York

E-ISSN 2196-9736

\section{Corresponding author}

\section{Masaaki Kobayashi, MD}

Department of

Gastroenterology and

Hepatology

Uonuma Institute of

Community Medicine

Niigata University Medical and Dental Hospital

4132 Urasa, Minami-Uonuma

Niigata 949-7302

Japan

Fax: +81-25-777-5067

masakoba@med.niigata-u.ac.jp
Background and study aims: We previously reported that narrow-band imaging with magnifying endoscopy (NBI-ME) revealed a unique "gastritis-like" appearance in approximately $40 \%$ of early gastric cancers after Helicobacter pylori eradication. Because rates of gastric cancer are increasing in patients with non-persistent infection of $H$. pylori, we aimed to clarify contribution factors to obscure tumors after therapeutic or spontaneous eradication.

Patients and methods: NBI-ME findings were examined retrospectively in 194 differentiated-type adenocarcinomas from $H$. pylori-negative patients with prior eradication therapy (83 patients) or without prior eradication therapy (72 patients). A gastritis-like appearance under NBI-ME was defined as an orderly microsurface structure and/or loss of clear demarcation with resemblance to the adjacent, non-cancerous mucosa. The correlation of this phenomenon with the degree of atrophic gastritis, determined both histo-

\section{Introduction \\ $\nabla$}

It is widely accepted that Helicobacter pylori is involved in the pathogenesis of gastritis and gastric cancer, and most gastric cancers do develop from $H$. pylori-infected gastric mucosa [1]. Recently, the use of $H$. pylori eradication therapy has spread worldwide [2,3], and the Japanese health insurance system approved eradication therapy in patients with $H$. pylori gastritis in 2013. Although eradication is expected to help prevent the development of gastric cancer in these patients, tumors have sometimes been discovered after successful eradication [4-6]. The characteristics and management of these cancers have become a major clinical issue [7-9], and it remains controversial whether $H$. pylori eradication can improve or interfere with the detection or diagnosis of gastric cancer. In a previous report, Ito et al. showed that $33 \%$ of gastric adenomas and cancers become logically in the adjacent mucosa and endoscopically, was evaluated.

Results: The tumor-obscuring gastritis-like appearance was observed in $42 \%$ and $23 \%$ of the patients in the $H$. pylori eradication and non-eradication groups, respectively. The development of this appearance was affected by the histological grade of atrophy $(P=0.003)$ and intestinal metaplasia $(P<0.001)$ on univariate analysis. Multivariate analysis revealed an odds ratio of 0.25 (95\% confidence interval $0.10-0.61, P=0.002$ ) for an endoscopically severe extent of atrophy, independently of eradication therapy.

Conclusions: An endoscopically mild or moderate extent of atrophy is associated with a gastritislike appearance under NBI-ME in currently H. pylori-negative gastric cancers. Surveillance endoscopy should be performed carefully after successful eradication or spontaneous elimination of $H$. pylori, particularly in patients with non-severe atrophic background mucosa.

flattened and indistinct during a short-term follow-up period after eradication therapy [10]; a normal columnar epithelium covering the cancer could influence the reliability of the endoscopic diagnosis of gastric cancers. We also previously described surface differentiation in approximately $40 \%$ of gastric cancers after eradication with the use of Ki-67 immunohistochemistry [11]. These gastric cancers had a "gastritis-like" appearance on narrow-band imaging with magnifying endoscopy (NBI-ME), resembling adjacent, non-cancerous mucosa. Because a flattened and indistinct appearance could obscure the early detection or accurate diagnosis of gastric cancer during endoscopic surveillance, it is important to clarify risk factors for this indistinct appearance in patients who have gastric cancer and who vary in $H$. pylori infection status, which may be post-eradication or otherwise. 


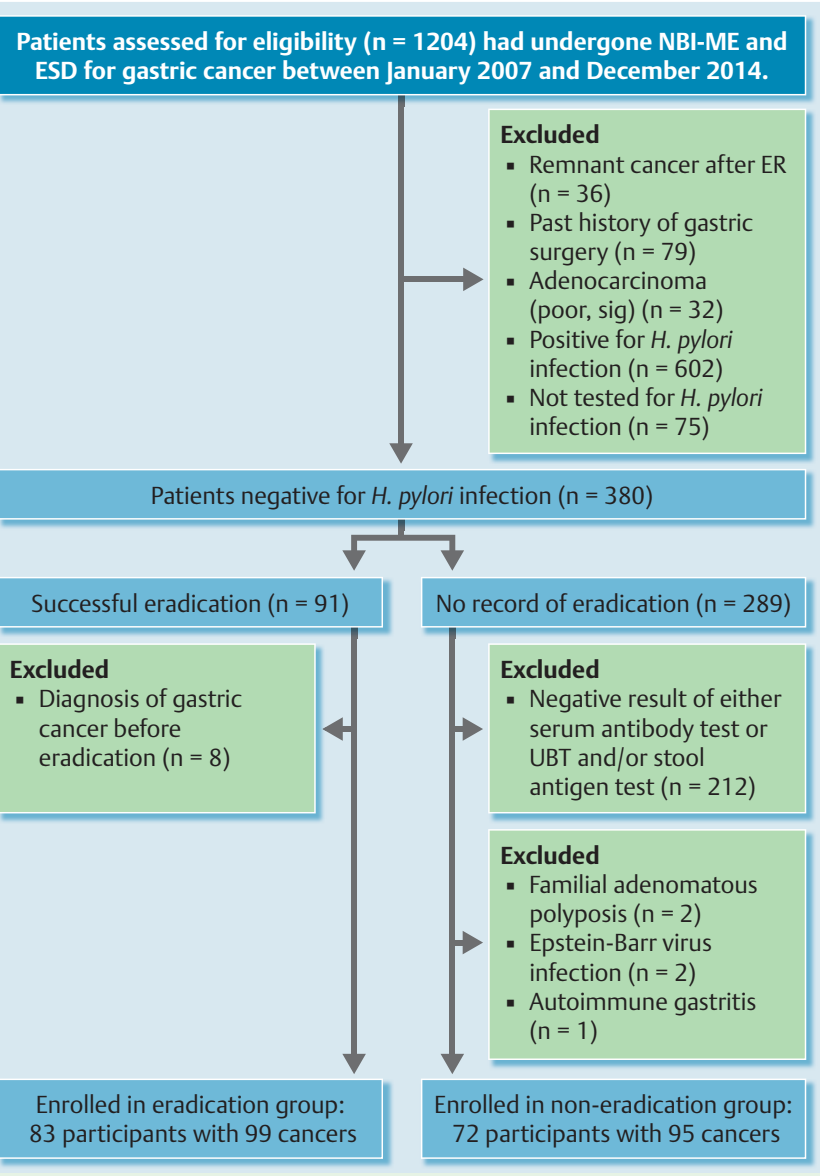

Fig. 1 Flowchart of patients and lesions included in a study of the effect of H. pylori eradication on NBI-ME of gastric tumors exhibiting non-severe extent of atrophy. NBI-ME, narrow-band imaging with magnifying endoscopy; ESD, endoscopic submucosal dissection; ER, endoscopic resection; poor, poorly differentiated adenocarcinoma; sig, signet ring cell adenocarcinoma; UBT, urea breath test.

Gastric cancers occasionally develop in patients who are not currently infected with $H$. pylori, irrespective of whether they have undergone prior eradication therapy. In recent reports, the prevalence of strictly defined $H$. pylori-uninfected gastric cancer was extremely low $(0.42 \%$ or $0.66 \%)$ [ 12,13$]$. Most patients who had gastric cancer without current $H$. pylori infection showed evidence of past infection [14], and as the rates of $H$. pylori infection decrease in the general population as well as in the elderly, the diagnosis of gastric cancer in patients with past $H$. pylori infection is likely to increase in clinical practice. However, it is unclear whether current diagnostic methods, including NBI-ME, can detect such gastric cancers. In the present study, we evaluated the NBI-ME and histological findings of currently $H$. pylori-negative gastric cancers in patients with and without a history of eradication therapy to determine the factors that affect the development of an indistinct gastritis-like appearance.

\section{Patients and methods}

$\nabla$

The clinical data of 1204 patients who had consecutively undergone endoscopic submucosal dissection (ESD) for early gastric cancer between January 2007 and December 2014 were collected at our hospital. All patients were scheduled to be fully examined under NBI-ME before ESD. The 36 patients who had undergone ESD of the remnant cancer after endoscopic resection and 79 who had undergone prior gastric surgery were excluded. The 32 patients with lesions representing predominantly poorly differentiated adenocarcinoma or signet ring cell adenocarcinoma were also excluded because these lesions are difficult to diagnose by NBI-ME regardless of $H$. pylori status [15]. There were 602 patients with persistent $H$. pylori infection as well as 75 without information about their $H$. pylori infection status, and these patients were also excluded. Patients were considered to be infected with $H$. pylori if a positive result was obtained after at least one of four tests: double-site biopsy culture, a carbon 13 urea breath test (UBT; Otsuka, Tokushima, Japan), a stool antigen test with an enzyme-linked immunosorbent assay (Premier Platinum HpSA; Meridian Bioscience, Cincinnati, Ohio, USA), and a serum immunoglobulin $\mathrm{G}$ antibody test (E-plate; Eiken Chemical Co., Tokyo, Japan).

Of the remaining 380 patients, who were negative for $H$. pylori infection, one group had undergone successful eradication therapy at our hospital, an affiliated hospital, or other clinics. The UBT and/or the stool antigen test were used to confirm successful eradication. The 8 patients in whom gastric cancer had been diagnosed before eradication were not included. A subpopulation of 42 patients had been involved in our prior study of NBI-ME of gastric cancer after eradication [11].

Patients in the non-eradication group were negative for $H$. pylori infection with no history of eradication therapy. To exclude patients with false-negative tests for $H$. pylori infection in this study, identification of non-persistent $H$. pylori infection was based on obtaining negative results in both the serum antibody test and the UBT and/or stool antigen test; 212 patients who did not meet the strictly defined criteria for non-persistent $H$. pylori infection were excluded. To analyze the influence of $H$. pylori status, 2 patients with familial adenomatous polyposis, 2 patients with Epstein-Barr virus infection, and 1 patient with autoimmune gastritis were excluded. Finally, an eradication group of 99 gastric cancers in 83 patients and a non-eradication group of 95 cancers in 72 patients were enrolled and reviewed retrospectively ( $\bullet$ Fig. 1 ). The study protocol was reviewed and approved by the ethics committee of our institution. Written informed consent was obtained from all patients before enrollment.

\section{Endoscopic procedure}

The instruments used in the present study were a magnifying video endoscope and an electronic endoscopic system (GIFH260Z Evis Lucera or Evis Lucera Elite Spectrum; Olympus Medical Systems, Tokyo, Japan). As previously reported [16], NBI-ME examinations and recordings of endoscopic findings were undertaken by four highly experienced endoscopists (M. K., S.H., K. M., and M.T.). The structural enhancement level was set at B8 for the NBI mode. The ESD procedure was completed, and the tumors were resected en bloc in all cases. Three representative cases in which the patients had undergone NBI-ME, ESD, and pathologic evaluation for early gastric cancer are shown in $\bullet$ Figs.2-4.

\section{Endoscopic evaluation}

NBI-ME criteria for gastric cancers were based on the microvascular architecture and/or microsurface structure with a clear demarcation between the cancerous and surrounding non-cancerous areas, as in previous reports [15]. Even in H. pylori-negative cancers, the criteria for a conspicuous NBI-ME appearance are defined by irregular, varied, and asymmetric crypt epithelium 

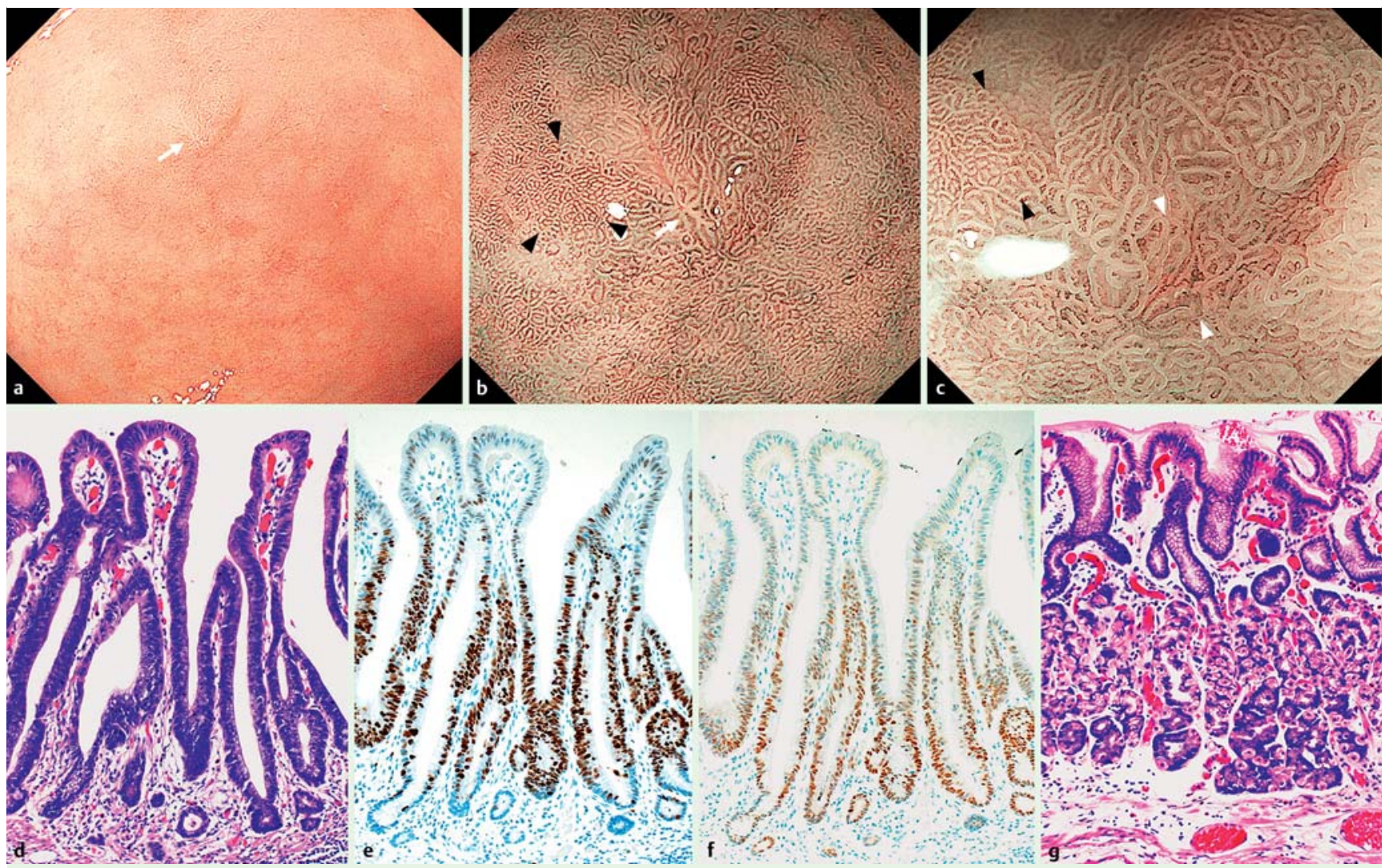

Fig. 2 Differentiated-type early gastric cancer was detected in patients who were not currently infected with $\mathrm{H}$. pylori without history of eradication therapy. a Conventional endoscopic findings of atrophic mucosa in the lessor curvature of the antrum. The lesion cannot be clearly recognized near the biopsy scar (white arrow). b Narrow-band imaging with magnified endoscopy (NBI-ME, low grade magnification). The lesion is a brownish, depressed-type area near the biopsy scar (white arrow, corresponds to a), and the surrounding mucosal area shows a regular pit microstructure (black arrowheads). c "Gastritis-like" appearance under NBI-ME (high grade magnification), characterized by a microsurface structure consisting of mixed papillae and grooves, resembling the surrounding non-cancerous mucosa without clear demarcation. Arrowheads indicate slice borders of the sections shown in $\mathbf{d}$, $\mathbf{e}$, and $\mathbf{f}$ (white) and in $\mathbf{g}$ (black). d Well-differentiated tubular adenocarcinoma with low grade atypia and an intertubular projection at the luminal surface (hematoxylin and eosin [H\&E], corresponds to white arrowheads in c). e Ki-67-positive cells located in the middle of the cancer tubules show surface differentiation. $\mathbf{f}$ P53-positive cells are scattered among the lower sections of the cancer tubules without a distinct boundary. $\mathbf{g}$ The conserved oxyntic glands in the surrounding non-cancerous mucosa (H\&E, corresponds to black arrowheads in c). The histological grade was estimated as inflammation (1), activity (0), atrophy (1), and intestinal metaplasia (0) according to the visual analogue scale in the updated Sydney system.

structures, as well as by irregular or absent microvessels. A demarcation line is present for the entire lesion $(\bullet$ Fig. $4 \mathbf{b}-\mathbf{d})$. NBI-ME findings of a gastritis-like appearance were characterized by uniform papillae and/or tubular pits bordered by clear "white zones" at the periphery, and by regular or faint microvessels [11]. White zones appeared as semi-translucent linear areas and were visualized by backscatter of the light under NBI [17]. Because the microsurface and microvascular features resembled those of the surrounding non-cancerous mucosa, the demarcations were not clear in parts of the lesion or sometimes throughout. In this study, the NBI-ME diagnosis of a gastritis-like appearance was determined based on the presence of an orderly microsurface structure and/or the loss of a clear demarcation line ( $\bullet$ Figs. 2c, 3d). Final determination of a gastritis-like appearance was according to the consensus of two highly experienced endoscopists (M.K. and S.H.), who independently performed blinded evaluations of the histological features.

The endoscopic extent of atrophy was classified according to the system of Kimura and Takemoto (closed-1 to open-3) [18], in which closed- 1 and closed- 2 are considered mild, closed- 3 and open- 1 as moderate, and open- 2 and open- 3 as severe. The endoscopic data for atrophy were obtained from the endoscopy re- ports of the clinical practice. Lesions lacking evidence of atrophic change (i.e., the observation of a regular arrangement of collecting venules at the lesser curvature of the lower gastric body on endoscopy [19]) were classified as closed-0 to distinguish them from closed-1. In addition to the endoscopic evaluation of mucosal atrophy, serum pepsinogen levels (PG I and PG II) were analyzed by Bio Medical Laboratories (Tokyo, Japan). When the two criteria of a PG I level of $70 \mathrm{ng} / \mathrm{mL}$ or lower and a ratio of PG I to PG II of 3.0 or lower were simultaneously fulfilled, the serum PG status was defined as positive, indicating the presence of atrophic gastritis [20]. Because $H$. pylori eradication therapy decreases the values of serum PG I and PG II and increases the ratio of PG I to PG II [21,22], the PG test was performed only in the non-eradicated group.

\section{Pathological evaluation}

The pathological findings for location, histological type, macroscopic type, and depth of invasion were reviewed according to the Japanese [23] and World Health Organization [24] classifications for gastric carcinoma. In this study, intraepithelial neoplastic lesions with low and/or high grade cellular and architectural 


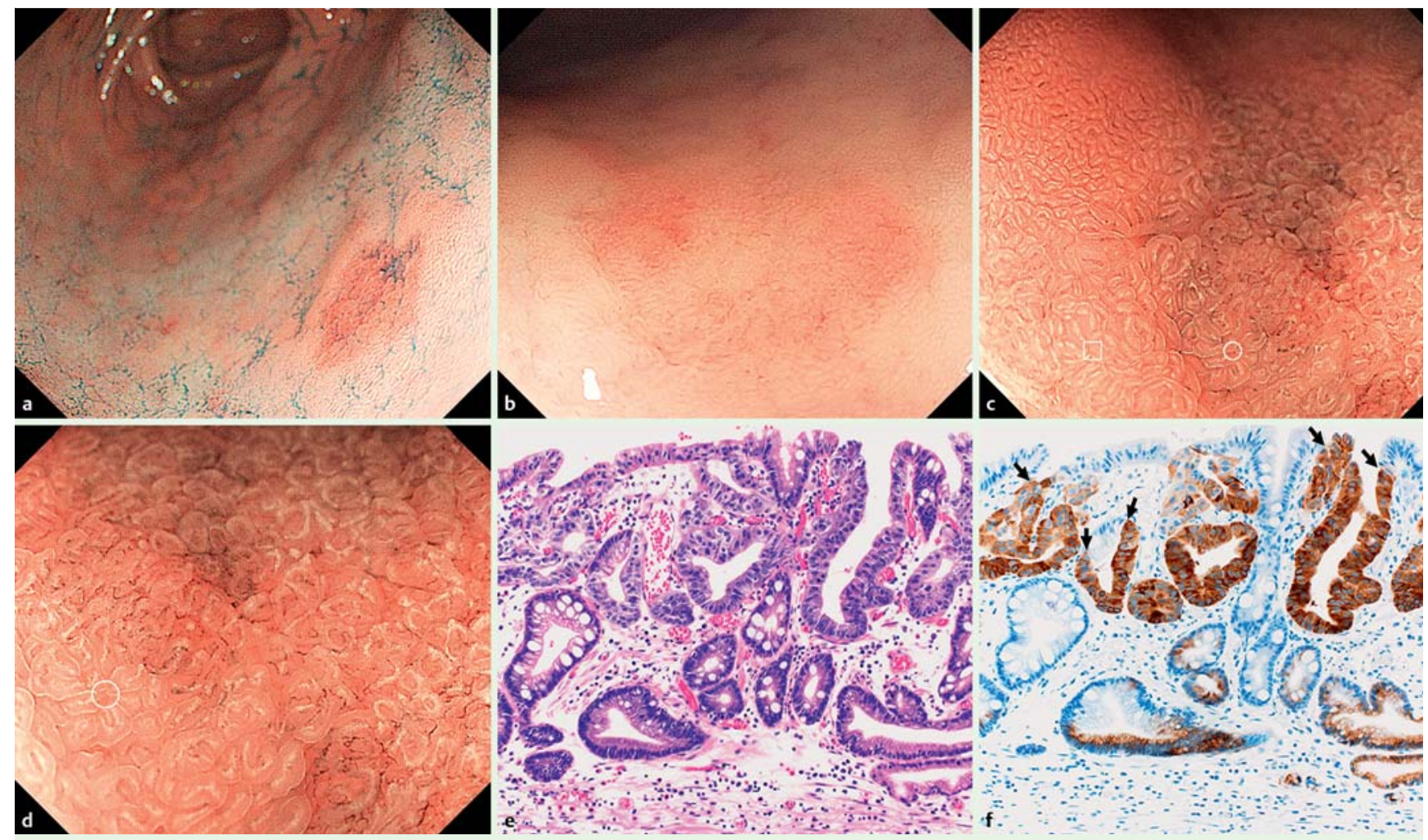

Fig. 3 Early, differentiated-type early gastric cancer detected after successful $H$. pylori eradication. a, b Conventional endoscopic and chromoendoscopic findings. The reddish, depressed-type lesion is located in the greater curvature of the antrum. $\mathbf{c}, \mathbf{d}$ Narrow-band imaging with magnified endoscopy shows a "gastritis-like" appearance in which a microsurface structure (circle) comprising regular papillae with a white opaque substance resembles the adjacent noncancerous mucosa (square). e Well-differentiated tubular adenocarcinoma with high grade atypia (hematoxylin and eosin). $\mathrm{f}$ Immunohistochemical staining demonstrates a predominantly gastric phenotype. The carcinoma cells are strongly positive for MUC6. The superficial non-neoplastic epithelium is interspersed among and above the cancer tubules and is characterized by negative staining for MUC6, showing several boundaries (black arrows).

atypia were categorized as non-invasive intramucosal carcinoma ( $\bullet$ Figs. $2 \mathrm{~d}, 3 \mathrm{e}, \mathbf{4 e}$ ).

The histological grades of mononuclear cell inflammation (inflammation), neutrophil infiltration (activity), glandular atrophy (atrophy), and intestinal metaplasia were estimated in the nonneoplastic mucosa adjacent to the cancer by using the samples taken at ESD. Because atrophy and intestinal metaplasia could be either diffuse or multifocal $[25,26]$, the histological grade was evaluated in the surrounding mucosal area (100 microscopic fields) showing the lowest grade of atrophy $(\bullet$ Fig. $\mathbf{2 g}$ ) and determined to be normal, mild, moderate, or marked according to the visual analogue scale in the updated Sydney system [26].

To determine the histological findings of surface differentiation and superficial non-neoplastic epithelium on top of or interspersed with the cancer, cellular proliferation and mucin phenotyping of the lesions with an indistinct gastritis-like appearance were assessed with immunohistochemistry for Ki-67, P53, and mucin markers (MUC5AC, MUC6, MUC2, CD10, and CDX2). Immunohistochemical staining was carried out as previously described $[11,16]$. Surface differentiation was regarded as positive when Ki-67-positive cells were restricted to the middle or lower layer of the lesion ( $\bullet$ Fig. 2 e); care was taken to exclude any interposed non-neoplastic epithelium [11]. Superficial non-neoplastic epithelium over more than one-third of the cancer tubules was determined by the difference in immunopositivity for Ki-67, P53, or mucin markers as well as histological cellular atypia (๑ Fig.3f).

\section{Statistical analysis}

The clinicopathological data for the patients and lesions were analyzed with a Mann-Whitney $U$ test for numerical data and a chisquared test or Fisher's exact probability test for categorical data. Interobserver agreement for NBI-ME diagnosis was estimated with $\mathrm{K}$ values. Multiple logistic regression analysis with forward stepwise model selection was used to explore the factors contributing to the indistinct gastritis-like appearance. Odds ratios and $95 \%$ confidence intervals were estimated. All analyses were performed with IBM SPSS Statistics for Windows, version 21 (IBM, Armonk, New York, USA). P values below 0.05 were considered significant.

\section{Results \\ $\nabla$}

\section{Baseline characteristics of patients}

The clinical data of the patients in the $H$. pylori eradication and non-eradication groups are summarized in $\bullet$ Table 1 . Significant differences in age, sex, and endoscopic extent of mucosal atrophy were found between the two groups. The number of older and female patients was larger in the non-eradication group than in the eradication group, and the patients in the non-eradication group had a greater extent of mucosal atrophy. Nevertheless, a considerable number of patients in both groups had a mild extent of mucosal atrophy. There were 2 patients in the non-eradication group with a classification of closed- 0 . The patients with positive PG test results had a severe $(n=22,79 \%)$ or moderate $(n=5,18 \%)$ 

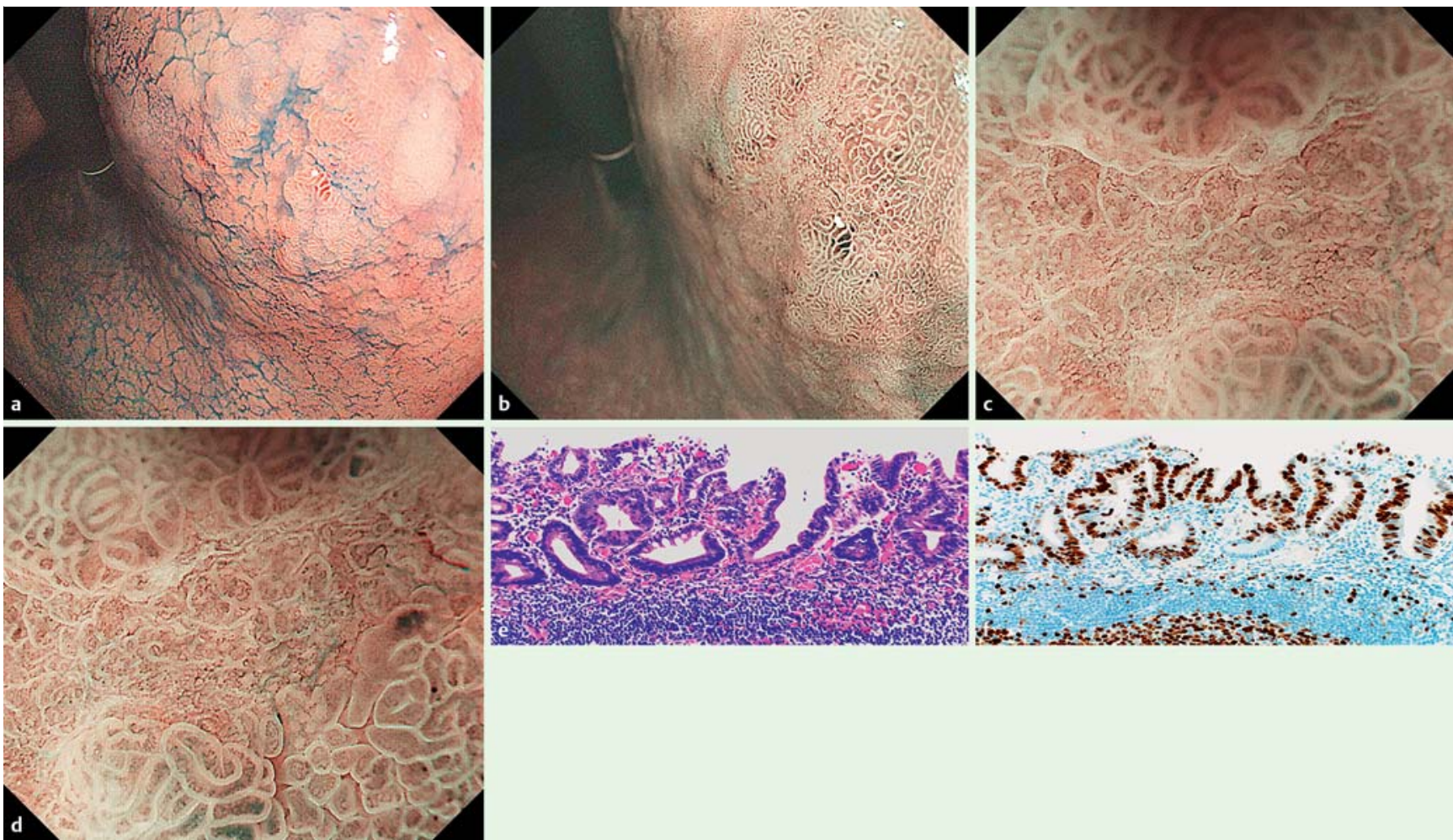

Fig. 4 Differentiated-type early gastric cancer detected after successful $\mathrm{H}$. pylori eradication. a Chromoendoscopy. The depressed-type lesion is located in the posterior wall of the body. $\mathbf{b}$ Narrow-band imaging with magnified endoscopy (NBI-ME, low grade magnification). The lesion is a depressed area; the surrounding mucosal area exhibits a groove microstructure. c, $\mathbf{d}$ Conspicuous appearance of gastric cancer under NBI-ME (high grade magnification) is characterized by an irregular, varied, and asymmetric microstructure bordered by an irregular white zone and irregular microvessels. A clear demarcation is seen between the cancer and the surrounding non-cancerous area. e Well-differentiated tubular adenocarcinoma with low grade atypia (hematoxylin and eosin). f Ki-67-positive cells are located in the top of the cancer tubules.

extent of atrophy. Target lesions were detected within 12 months after eradication therapy in 12 patients who underwent endoscopic resection for the initial gastric cancer and in 9 patients treated for peptic ulcers.

\section{Clinicopathological characteristics of gastric cancer according to NBI-ME findings}

In terms of NBI-ME diagnosis, the indistinct, gastritis-like appearance was observed in 42 of 99 cancers in the eradication group ( $42 \%$ ) and in 22 of 95 cancers in the non-eradication group $(23 \%)$. The concordance of the two endoscopists' assessments of the NBI-ME findings was $87 \%(169 / 194)$, and the level of interobserver agreement was graded as substantial $(\mathrm{K}=0.69)$. We used univariate analysis to examine the clinicopathological factors associated with the observation of a gastritis-like appearance under NBI-ME $(\bullet$ Table 2$)$. A gastritis-like appearance was significantly correlated with an endoscopically mild or moderate extent of atrophy in all lesions $(P<0.001)$. The histological tub1 type, with low-grade atypia, was observed frequently in lesions with a gastritis-like appearance $(P<0.001)$. The gastritis-like appearance was significantly correlated with the histological findings of surface differentiation and superficial non-neoplastic epithelium $(P<0.001)$. No significant differences were noted for size, macroscopic type, or depth of invasion.

The histological grade of inflammation and activity in the nonneoplastic mucosa adjacent to the cancer according to the visual analogue scale in the updated Sydney system was not correlated with the NBI-ME findings in either group. However, the histological grades of atrophy and intestinal metaplasia were significantly
Table 1 Baseline characteristics of patients with currently H. pylori-negative gastric cancers.

\begin{tabular}{|c|c|c|}
\hline & $\begin{array}{l}\text { Eradication } \\
\text { group } \\
\text { (83 patients) }^{1}\end{array}$ & $\begin{array}{l}\text { Non-eradication } \\
\text { group } \\
\text { (72 patients) }^{1}\end{array}$ \\
\hline Age, $y^{2}$ & $70.0(38-81)$ & $77.0(54-88)$ \\
\hline \multicolumn{3}{|l|}{$\operatorname{Sex}^{3}$} \\
\hline Male & $69(83)$ & $44(61)$ \\
\hline Female & $14(17)$ & $28(39)$ \\
\hline \multicolumn{3}{|l|}{ Extent of mucosal atrophy $y^{3,4}$} \\
\hline Closed-1, closed-2 & $12(14)$ & $13(18)$ \\
\hline Closed-3, open-1 & $47(57)$ & $16(22)$ \\
\hline Open-2, open-3 & $24(29)$ & $43(60)$ \\
\hline \multicolumn{3}{|l|}{ Pepsinogen test for atrophy ${ }^{5}$} \\
\hline Positive & - & $28(39)$ \\
\hline Negative & - & $44(61)$ \\
\hline \multicolumn{3}{|l|}{ Primary disease to eradication } \\
\hline Gastric or duodenal ulcer & $35(42)$ & - \\
\hline GC, post-ER & $38(46)$ & - \\
\hline Others & $10(12)$ & - \\
\hline Duration after eradication, mo & $48.0(3-247)$ & - \\
\hline
\end{tabular}

GC, gastric cancer; ER, endoscopic resection.

${ }^{1}$ Data are expressed as number (percent) or median (range)

$2 p<0.001$

$3 P<0.01$.

${ }^{4}$ Extent of mucosal atrophy was determined according to the Kimura-Takemoto classification [18]

${ }^{5}$ The pepsinogen (PG) test result was considered positive when the two criteria of PG I level of $70 \mathrm{ng} / \mathrm{mL}$ or lower and ratio of PG I to PG II of 3.0 or lower were simultaneously fulfilled. 
Table 2 Correlation of clinicopathological characteristics of currently $\mathrm{H}$. pylori-negative gastric cancers with NBI-ME appearance.

\begin{tabular}{|c|c|c|c|}
\hline \multirow[b]{2}{*}{ Characteristic } & \multicolumn{3}{|l|}{ NBI-ME appearance } \\
\hline & Gastritis-like (64 lesions) & Conspicuous (130 lesions) & $P$ value \\
\hline History of eradication therapy: positive/negative & $42 / 22$ & $57 / 73$ & 0.006 \\
\hline Endoscopic extent of atrophy: mild, moderate/severe ${ }^{1}$ & $50 / 14$ & $55 / 75$ & $<0.001$ \\
\hline Size: $\leq 10 \mathrm{~mm} />10 \mathrm{~mm}$ & $29 / 35$ & $55 / 75$ & 0.758 \\
\hline \multicolumn{4}{|l|}{ Location: } \\
\hline upper, middle/lower & $43 / 21$ & $67 / 63$ & 0.046 \\
\hline less/gre, ant, post & $34 / 30$ & $65 / 65$ & 0.760 \\
\hline Macroscopic type: elevated/depressed & $22 / 42$ & $42 / 88$ & 0.871 \\
\hline Histological type, predominant: tub1 (low)/tub1 (high), tub2 & $51 / 13$ & $68 / 62$ & $<0.001$ \\
\hline Depth of invasion: mucosa/submucosa & $58 / 6$ & $117 / 13$ & 1.000 \\
\hline Surface differentiation: positive/negative & $39 / 25$ & $15 / 115$ & $<0.001$ \\
\hline Non-neoplastic epithelium: positive/negative & $26 / 38$ & $17 / 113$ & $<0.001$ \\
\hline Histological grade of atrophy: normal, mild, moderate/marked² & $38 / 26$ & $47 / 83$ & 0.003 \\
\hline Histological grade of intestinal metaplasia: normal, mild, moderate/marked² & $58 / 6$ & $77 / 53$ & $<0.001$ \\
\hline
\end{tabular}

NBI-ME, narrow-band imaging with magnified endoscopy; less/gre, ant, post, lesser/greater curvature, anterior or posterior wall; tub1 (low/high), well-differentiated tubular adenocarcinoma, low/high grade atypia; tub2, moderately differentiated tubular adenocarcinoma.

${ }^{1}$ Endoscopic extent of atrophy was determined according to the Kimura-Takemoto classification [18]: mild, closed-1, closed-2; moderate, closed-3, open-1; severe, open-2, open-3.

${ }^{2}$ Histological grades of atrophy and intestinal metaplasia were evaluated in the surrounding mucosa adjacent to the cancer according to the updated Sydney system [26].

correlated with the NBI-ME findings $(P=0.003$ and $P<0.001$, respectively; 0 Table 2 ). In the eradication group, the length of time following eradication was not a significant factor. Serum PG status was significantly related to the NBI-ME appearance in the non-eradication group $(P<0.05)$; the PG status was more frequently positive in conspicuous lesions $(45 \%, 33 / 73)$ than in indistinct, gastritis-like lesions (18\%, 4/22).

Results of the multiple logistic regression analysis to determine factors independently contributing to a tumor-obscuring, gastritis-like appearance are shown in $\bullet$ Table 3 . The endoscopic extent of atrophy was significantly and independently correlated with a gastritis-like appearance. The correlations of the grades of histological atrophy and intestinal metaplasia, however, may depend on the endoscopic findings. Surface differentiation, superficial non-neoplastic epithelium, and histological type were factors that contributed significantly to an indistinct appearance.

\section{Discussion}

$\nabla$

In the present study of patients with non-persistent $H$. pylori infection, early, differentiated-type gastric cancers showed a gastritis-like appearance under NBI-ME in $42 \%$ of patients in the H. pylori eradication group and in $23 \%$ of those in the non-eradication group. The presence of mild or moderate atrophic mucosa on endoscopy was associated with the development of a gastritislike appearance in both groups. During endoscopic surveillance for early gastric cancer, the NBI-ME diagnosis of a gastritis-like appearance should be more carefully scrutinized in patients who are currently negative for $\mathrm{H}$. pylori-negative infection and have mild or moderate atrophic gastritis than in patients with severe atrophic gastritis, regardless of eradication history.

Because NBI-ME is a special modality for enhancing the visualization of microvessels and microstructure within the superficial layer of the gastric mucosa, it could be used to examine surface differentiation and superficial non-neoplastic epithelium because both exhibit a gastritis-like appearance. In $\bullet$ Fig. 3, for example, the white opaque substance is usually considered an intestinal phenotype marker [27], whereas the lesion has a gas-
Table 3 Multiple logistic regression analysis of factors associated with a gastritis-like appearance under narrow-band imaging with magnified endoscopy.

\begin{tabular}{|lllr|}
\hline Factor $^{1}$ & OR & 95\%Cl & P value \\
\hline Endoscopic extent of atrophy & 0.25 & $0.10-0.61$ & 0.002 \\
\hline Histological type & 0.23 & $0.09-0.63$ & 0.004 \\
\hline Surface differentiation & 8.89 & $3.67-21.6$ & $<0.001$ \\
\hline Non-neoplastic epithelium & 5.64 & $2.08-15.3$ & 0.001 \\
\hline
\end{tabular}

$\mathrm{OR}$, odds ratio; $\mathrm{Cl}$, confidence interval.

1 Significant variables were selected by using forward stepwise regression.

tric mucin phenotype immunohistochemically. Therefore, a microsurface structure containing the white opaque substance reflects the presence of superficial non-neoplastic epithelium on the tumor and also on the surrounding non-cancerous mucosa. Multivariate analysis revealed that a gastritis-like appearance under NBI-ME was independently correlated with non-severe endoscopic atrophy as well as surface differentiation and superficial non-neoplastic epithelium. In our prior study [11], we demonstrated a gastritis-like appearance under NBI-ME in 22 gastric cancers following eradication. Patients with these gastric cancers showed a tendency toward a mild or moderate extent of atrophy, but the differences were not significant $(P=0.088)$, probably owing to the small sample size. In this study, a gastritis-like appearance was observed under NBI-ME in 42 lesions in the eradication group; the correlation between a gastritis-like appearance and the endoscopic extent of atrophy was significant in our case $(P=$ 0.007).

Building upon our prior study, we examined patients with a currently H. pylori-negative status and without a history of eradication therapy to determine whether the endoscopic extent of atrophy affected the development of a gastritis-like appearance. The difficulty of distinguishing positivity for past $H$. pylori infection in gastric mucosa from stronger atrophic changes is widely acknowledged. In this study, patients currently negative for $\mathrm{H}$. $\mathrm{py}$ lori infection were selected based on negative results of both a serum antibody test and a UBT and/or stool antigen test, with care taken to exclude patients with false-negative results for $\mathrm{H}$. pylori 
infection. A large extent of atrophy was observed in $60 \%$ of patients in the non-eradication group ( $\bullet$ Table 1 ). A past infection is difficult to identify in these patients; however, $H$. pylori may have been spontaneously eliminated when the mucosa changed from atrophic to metaplastic, given that $H$. pylori cannot survive in metaplastic mucosa [28]. NBI-ME revealed a conspicuous appearance in 52 of 60 gastric cancers (87\%) associated with severe atrophic mucosa in the non-eradication group.

Conversely, 29 of the 72 patients in the non-eradication group (40\%) were $H$. pylori-negative but had mild or moderate atrophy ( Table1). The reason for the spontaneous elimination of $H$. pylori in these patients was unclear. Although we could not confirm a history of eradication therapy, it is possible that a misunderstanding occurred in communications with the patients concerning previous eradication therapy, perhaps owing to insufficient explanations from their chief physicians. These patients may also have undergone unplanned $H$. pylori eradication because antibiotics are commonly used to treat other diseases in Japan. NBIME revealed a gastritis-like appearance in 14 of 35 gastric cancers with a mild or moderate extent of atrophic mucosa (40\%) in the non-eradication group. The prevalence of the gastritis-like appearance was similar to that observed in the eradication group. Therefore, mild or moderate mucosal atrophy was associated with the development of the gastritis-like appearance irrespective of eradication history.

Previous studies revealed that the risk factors for the development of primary or secondary cancer after eradication include advanced atrophic change in the gastric corpus [7] and intestinal metaplasia $[29,30]$. However, less advanced atrophic change may be a risk factor for failure to detect cancer. Patients with closed-0 type lesions may theoretically be free of $H$. pylori, and 1 these 2 patients had indistinct cancers revealed under NBI-ME. The prevalence of gastric cancer not infected with $H$. pylori was extremely low $(0.69 \%, 2 / 289)$ in candidates with no $H$. pylori infection and no record of eradication.

The mechanism underlying the correlation of a gastritis-like appearance with mild atrophic change is unknown. We confirmed that this NBI-ME finding was correlated with the histological grade of atrophy and intestinal metaplasia in non-neoplastic mucosa adjacent to the cancer. Thus, we considered the possibility that a tumor located at markedly atrophic mucosa with obvious intestinal metaplasia might resemble an ordinary gastric cancer in appearance. In contrast, an indistinct cancer would be accompanied by mild or moderate atrophic mucosa that might be less atrophic after therapeutic (or spontaneous) eradication. Based on pathological investigations, Correa indicated that patchy areas of atrophic-metaplastic changes in the mucosa of the antrum or corpus (i.e., multifocal atrophic gastritis) frequently coexists with gastric cancer [25]. Kanzaki et al. revealed that mucosa with chronic gastritis in the corpus exhibited either of two types of microscopic mucosal structures under NBI-ME: a foveolar type with low grade atrophy and intestinal metaplasia and a groove type with high grade atrophy and intestinal metaplasia [31]. Groove-type mucosa occurs focally even in a mild extent of endoscopic atrophy, whereas foveolar-type mucosa is common in a moderate extent of atrophy ( $\bullet \mathbf{F i g .} \mathbf{2} \mathbf{b}, \mathbf{g}$ ). In a recent prospective follow-up study after $H$. pylori eradication, Kodama et al. confirmed that the histological grade of atrophy markedly decreases in the greater curvature of the corpus after eradication except in patients with severe (open-3) endoscopic atrophy before eradication [32]. Thus, gastric cancers may develop in a non-severe extent of atrophic gastritis before eradication and may be indistin- guishable from the surrounding mucosa, which becomes less atrophic after eradication. Further time-dependent studies are necessary to clarify the influence of $H$. pylori elimination on surface differentiation and superficial non-neoplastic epithelium, together with repaired atrophy of the surrounding mucosa.

There are limitations to be considered in this cross-sectional study. It was conducted at a single center, and the images acquired via NBI-ME were reviewed retrospectively for evidence of background atrophic mucosa. Multicenter, prospective, and realtime diagnostic studies are required to further evaluate the significance of a gastritis-like appearance on NBI-ME and its relationship with the extent of atrophic change.

\section{Conclusion \\ $\nabla$}

In this study, we demonstrated that gastric cancer is often found by endoscopic examination in patients with no current $H$. pylori infection. Mild or moderate atrophy in the background mucosa correlated with a tumor-obscuring, gastritis-like appearance on NBI-ME. Surveillance endoscopy should be performed carefully after the successful eradication or spontaneous elimination of $H$. pylori infection, particularly in patients with a decrease in mucosal atrophy.

\section{Competing interests: None}

\section{Institutions}

${ }^{1}$ Department of Gastroenterology and Hepatology, Uonuma Institute of Community Medicine, Niigata University Medical and Dental Hospital, Niigata, Japan

2 Division of Gastroenterology and Hepatology, Niigata University Graduate School of Medical and Dental Sciences, Niigata, Japan

${ }^{3}$ Division of Molecular and Diagnostic Pathology, Niigata University Graduate

School of Medical and Dental Sciences, Niigata, Japan

${ }^{4}$ Department of Medical Informatics, Niigata University Graduate School of Medical and Dental Sciences, Niigata, Japan

\section{References}

1 Uemura N, Okamoto S, Yamamoto $S$ et al. Helicobacter pylori infection and the development of gastric cancer. N Engl J Med 2001; 345: $784-$ 789

$2 W u C Y, K u o K N, W u$ MS et al. Early Helicobacter pylori eradication decreases risk of gastric cancer in patients with peptic ulcer disease. Gastroenterology 2009; 137: 1641 - 1648

3 Asaka M, Kato M, Takahashi S et al. Guidelines for the management of Helicobacter pylori infection in Japan: 2009 revised edition. Helicobacter 2010; 15: $1-20$

4 Take S, Mizuno M, Ishiki K et al. The effect of eradicating Helicobacter pylori on the development of gastric cancer in patients with peptic ulcer disease. Am J Gastroenterol 2005; 100: 1037-1042

5 Fukase K, Kato M, Kikuchi S et al. Effect of eradication of Helicobacter pylori on incidence of metachronous gastric carcinoma after endoscopic resection of early gastric cancer: an open-label, randomised controlled trial. Lancet 2008; 372: $392-397$

6 Mabe K, Takahashi M, Oizumi H et al. Does Helicobacter pylori eradication therapy for peptic ulcer prevent gastric cancer? World J Gastroenterol 2009; 15: 4290-4297

7 Kamada T, Hata J, Sugiu K et al. Clinical features of gastric cancer discovered after successful eradication of Helicobacter pylori: results from a 9-year prospective follow-up study in Japan. Aliment Pharmacol Ther 2005; 21: $1121-1126$

8 Ito $M$, Takata $S$, Tatsugami $M$ et al. Clinical prevention of gastric cancer by Helicobacter pylori eradication therapy: a systematic review. J Gastroenterol 2009; 44: 365 - 371

9 Yamamoto K, Kato M, Takahashi M et al. Clinicopathological analysis of early-stage gastric cancers detected after successful eradication of Helicobacter pylori. Helicobacter 2011; 16: 210-216 
10 Ito M, Tanaka S, Takata S et al. Morphological changes in human gastric tumours after eradication therapy of Helicobacter pylori in a shortterm follow-up. Aliment Pharmacol Ther 2005; 21: 559-566

11 Kobayashi M, Hashimoto S, Nishikura K et al. Magnifying narrow-band imaging of surface maturation in early differentiated-type gastric cancers after Helicobacter pylori eradication. J Gastroenterol 2013; 48: $1332-1342$

12 Matsuo T, Ito $M$, Takata $S$ et al. Low prevalence of Helicobacter pylorinegative gastric cancer among Japanese. Helicobacter 2011; 16: 415419

13 Ono S, Kato M, Suzuki M et al. Frequency of Helicobacter pylori-negative gastric cancer and gastric mucosal atrophy in a Japanese endoscopic submucosal dissection series including histological, endoscopic and serological atrophy. Digestion 2012; 86: 59-65

14 Kwak HW, Choi IJ, Cho SJ et al. Characteristics of gastric cancer according to Helicobacter pylori infection status. J Gastroenterol Hepatol 2014; 29: 1671 - 1677

15 Yao K, Anagnostopoulos GK, Ragunath K. Magnifying endoscopy for diagnosing and delineating early gastric cancer. Endoscopy 2009; 41: $462-467$

16 Kobayashi M, Takeuchi M, Ajioka Yet al. Mucin phenotype and narrowband imaging with magnifying endoscopy for differentiated-type mucosal gastric cancer. J Gastroenterol 2011; 46: 1064 - 1070

17 Yagi K, Nozawa Y, Endou $S$ et al. Diagnosis of early gastric cancer by magnifying endoscopy with NBI from viewpoint of histological imaging: mucosal patterning in terms of white zone visibility and its relationship to histology. Diagn Ther Endosc 2012; 2012: 954809

18 Kimura K, Takemoto T. An endoscopic recognition of the atrophic border and its significance in chronic gastritis. Endoscopy 1969; 1: 87-97

19 Yagi K, Nakamura A, Sekine A. Characteristic endoscopic and magnified endoscopic findings in the normal stomach without Helicobacter pylori infection. J Gastroenterol Hepatol 2002; 17: 39-45

20 Samloff IM, Varis K, Ihamaki $T$ et al. Relationships among serum pepsinogen I, serum pepsinogen II and gastric mucosal histology. A study in relatives of patients with pernicious anemia. Gastroenterology 1982; 83: 204-209

21 Furuta T, Kaneko E, Baba S et al. Percentage changes in serum pepsinogens are useful as indices of eradication of Helicobacter pylori. Am J Gastroenterol 1997; 92: 84-88
22 Ohkusa T, Takashimizu I, Fujiki K et al. Changes in serum pepsinogen, gastrin, and immunoglobulin $\mathrm{G}$ antibody titers in Helicobacter pyloripositive gastric ulcer after eradication of infection. J Clin Gastroenterol 1997; 25: 317-322

23 Japanese Gastric Cancer Association. Japanese classification of gastric carcinoma. 3rd English edn. Gastric Cancer 2011; 14: 101 -112

24 Lauwers GY, Carneiro F, Graham DY et al. Gastric carcinoma. In: Bosman FT, Carneiro F, Hruban RH et al., eds. WHO classification of tumours of the digestive system. 4th edn. Lyon, France: IARC Press; 2010: 48-58

25 Correa P. Chronic gastritis: a clinic-pathological classification. Am J Gastroenterol 1988; 83: 504-509

26 Dixon MF, Genta RM, Yardley JH et al. Classification and grading of gastritis. The updated Sydney System. International Workshop on the Histopathology of Gastritis, Houston 1994. Am J Surg Pathol 1996; 20: $1161-1181$

27 Yao K, Iwashita A, Nambu M et al. Nature of white opaque substance in gastric epithelial neoplasia as visualized by magnifying endoscopy with narrow-band imaging. Dig Endosc 2012; 24: 419-425

28 Rugge M, Di Mario F, Cassaro $M$ et al. Pathology of the gastric antrum and body associated with Helicobacter pylori infection in non-ulcerous patients: is the bacterium a promoter of intestinal metaplasia? Histopathology 1993; 22: 9-15

29 Kodama M, Murakami K, Okimoto T et al. Histological characteristics of gastric mucosa prior to Helicobacter pylori eradication may predict gastric cancer. Scand J Gastroenterol 2013; 48: 1249-1256

30 Shichijo S, Hirata Y, Sakitani K et al. Distribution of intestinal metaplasia as a predictor of gastric cancer development. J Gastroenterol Hepatol 2015; 30: $1260-1264$

31 Kanzaki $H$, Uedo $N$, Ishihara $R$ et al. Comprehensive investigation of areae gastricae pattern in gastric corpus using magnifying narrow band imaging endoscopy in patients with chronic atrophic fundic gastritis. Helicobacter 2012; 17: 224-231

32 Kodama M, Okimoto T, Ogawa R et al. Endoscopic atrophic classification before and after $\mathrm{H}$. pylori eradication is closely associated with histological atrophy and intestinal metaplasia. Endosc Int Open 2015; 3: E311-E317 